\title{
Intégrer la typographie et la ponctuation à l'enseignement/apprentissage du FLE
}

Guillaume Jeanmaire, Korea University, avec Daeyoung Kim, Chungbuk National University

\section{Introduction}

La typographie et la ponctuation ne semblent plus considérées avec autant d'importance aujourd'hui qu'autrefois, même au sein de l'enseignement. Outre qu'elles constituent une composante essentielle d'une langue, elles peuvent aussi en faciliter l'apprentissage. Mais compte tenu de la diversité des conventions d'une langue à une autre, il faut bien reconnaître qu'il est souvent difficile d'en faire apprécier les nuances. Les apprenants anglophones sont par exemple désarmés face à la typographie et à la ponctuation ${ }^{1}$ françaises, en particulier en raison des nombreuses différences entre l'anglais et le français, et ils ne sont pas les seuls, comme en témoignent les erreurs que nous avons fréquemment rencontrées auprès d'étudiants coréens ayant appris l'anglais en L2 depuis le collège, ainsi que d'étudiants d'échange anglophones canadiens et américains.

En effet, les apprenants d'Asie de l'Est (sinophones, japonophones, coréanophones) et les apprenants arabophones, dont la langue maternelle (LM) est typologiquement éloignée du français, et qui utilisent un autre système d'écriture que l'alphabet latin, prennent principalement ${ }^{2}$ comme repère l'anglais pour l'apprentissage d'une autre langue étrangère L3 comme le FLE (à ce sujet, voir Rozita Ilani ; Jeon-gil Han), l'anglais étant la langue L2 obligatoire et enseignée dès le plus jeune âge. L'anglais et le français recourant tous deux à l'alphabet latin, ces élèves présupposent que le français fonctionne comme l'anglais (Ilani 33 ; Han), en particulier pour les majuscules, dont leur LM est dépourvue.

Il faut bien constater que rares sont les manuels de FLE qui y consacrent un chapitre ou même qui abordent la question. Aider ces apprenants à surmonter leurs difficultés est notre objectif, qui s'inscrit dans le cadre de cours de FLE en les y sensibilisant dès le niveau débutant (niveaux A1 et A2 du CECR), puis en consolidant ces connaissances aux niveaux intermédiaire et avancé (à partir du niveau B1 du CECR). Le but de cet article n'est néanmoins ni de proposer un cours de ponctuation ou de typographie, ni d'en faire une revue exhaustive, mais de relier ces points au contenu du cours et à d'autres objectifs plus amples visant à développer toutes les compétences (langagières ou non), autrement dit de les enseigner en contexte, afin d'en faciliter l'ancrage. Cet article a pour objectif d'abord de mettre en évidence les difficultés que présente l'apprentissage de ces points, en insistant sur 
les interférences («transfert» négatif, Kellerman) entre anglais et français, et dans un deuxième temps de montrer la manière de les pallier. Comme le fait remarquer à juste titre cet auteur, le «transfert» de connaissances de la LM - et/ou d'une langue tierce comme l'anglais apprise antérieurement dans un contexte non anglophone - vers une langue étrangère (ici le FLE) ne se fera que si ces connaissances sont considérées comme universelles et donc adaptables par les apprenants. Plus que de connaître les différences entre deux systèmes linguistiques, ce qui importe est en effet de savoir comment appréhender et gérer les convergences et divergences entre anglais et français. L'auteur recourt au concept de «transfert» en le liant à la «psycho-typologie » des apprenants. Ainsi, il explique que le transfert ne se fera pas pour des éléments trop idiomatiques ou spécifiques, mais pour des éléments que les apprenants jugeront neutres («language neutral», Kellerman), moins marqués dans leur LM. Le transfert peut ainsi s'appliquer non seulement à des phénomènes lexicaux (ex. *Je suis *confortable dans ce *sofa < I am comfortable in this sofa), de graphie lexicale (ex. *marriage, *exercis $\underline{e}$ ), syntaxiques (ex. *Je souvent fais $<$ I often do) ou morphosyntaxiques (ex. place des pronoms, verbes transitifs en anglais mais intransitifs en français : ex. *on pardonne eux), mais aussi à la construction de catégories conceptuelles et grammaticales plus larges, autrement dit à un niveau conceptuel (typographie, majuscules, nom propre, nom commun, substantif, adjectif, nombre grammatical, etc.). Il apparaît dès lors utile de faire un «point » sur la ponctuation et de lui donner sa place dans les cours de FLE.

Parmi les difficultés que devront surmonter les apprenants, nous aborderons d'abord les écueils interférentiels liés successivement à la ponctuation et aux espaces, à la typographie et aux chiffres, aux majuscules et aux minuscules, puis d'autres difficultés typographiques relatives aux accents ou aux élisions obligatoires dont l'anglais est dépourvu, et enfin les problèmes que posent les traits d'union. Nous fournirons ensuite des pistes à partir du niveau débutant, en mettant en exergue les erreurs les plus récurrentes, en les intégrant à des objectifs plus vastes. Au niveau intermédiaire (niveaux B1 et B2 du CECR), nous verrons comment consolider ces connaissances dans le cadre d'ateliers de lecture orale et d'écriture. Enfin, au niveau avancé (à partir du niveau B2), nous proposerons de mettre en valeur les différences de ponctuation et de typographie entre l'anglais et le français à l'aide de corpus parallèles anglais-français (textes authentiques) et par la pratique de la traduction de textes de l'anglais vers le français. 


\section{2. Écueils interférentiels}

\subsection{Ponctuation et espaces}

Chez les apprenants anglophones (que l'anglais soit langue première L1 ou langue seconde L2), on rencontre des erreurs résultant d'interférences avec les règles typographiques de l'anglais. Par exemple, l'anglais, contrairement au français ${ }^{3}$, ne mettant pas d'espace devant les signes doubles de ponctuation (:; ! ?) ou à l'intérieur des guillemets, les erreurs foisonnent: ex. Pourquoi? au lieu de Pourquoi? Les tirets longs (dash) engendrent aussi régulièrement des écueils interférentiels, car, en français, on met une espace devant et derrière : texte - texte, contrairement à l'anglais (Ramat et Benoit 198, 214) : texte-texte. En début de ligne dans un dialogue, une espace est également de rigueur en français après le tiret (- texte). En outre, dans les dialogues, pour marquer le changement d'interlocuteur, l'utilisation des guillemets en anglais et celle des tirets (suivis d'une espace) en français diffèrent.

Par ailleurs, en anglais, figure à la fin d'une énumération une virgule avant $\underline{\underline{e t}}$; il n'est donc pas étonnant que les apprenants suivent l'exemple (ex. mon père, ma mère, et moi). Or, en français, la virgule ne s'utilise pas devant les conjonctions ou ou et lorsque celles-ci précèdent le dernier terme d'une énumération (Narjoux 46). De même, il n'est pas rare de relever, outre des majuscules superflues (cf. infra), des virgules superflues dans une date par imitation de l'anglais (Benoit et Ramat 213), notamment avant l'année : (...) aura lieu Lundi, Novembre 26, 2016 (< Monday, November 26 ${ }_{2}^{\text {th }}$ 2016) au lieu de lundi 26 novembre 2016. Inversement, les apprenants anglophones ne penseront pas d'eux-mêmes à apposer une virgule entre le chiffre et le mot dans une adresse (ex. 4, rue des Pontonniers), puisqu'il n'y en a pas en anglais (213).

A contrario, en anglais, on recourt moins souvent aux virgules en début de phrase, après notamment un complément circonstanciel de temps $($ Ce matin, ... ) ou autre marqueur logique ou temporel : ex. Cependant, ... L'absence de virgule après les adverbes de temps ou autre marqueur logique est donc fréquente. Comme le note Narjoux (49 ; voir aussi Grevisse et Goosse 156-163), plus généralement, la virgule apparaît fréquemment après des compléments adverbiaux ou circonstanciels (syntagmes ou propositions subordonnées circonstancielles) surtout lorsqu'ils sont situés en tête de phrase (ex. Derrière la porte, ... ; Quand il fait beau, ...). Les écueils interférentiels liés à la ponctuation et à la typographie sont d'ailleurs légion lors du passage de l'anglais vers le français ou inversement comme l'illustrent Claude Demanuelli et le chapitre V de Michel Ballard «Typographie et ponctuation ». À ce sujet, Ballard observe que les textes anglais sont beaucoup moins 
ponctués que les textes français. Les points virgules, tirets, parenthèses, points, points d'exclamation et surtout virgules s'avèrent bien plus fréquents en français qu'en anglais, d'où un ajout nécessaire de ces derniers lors du passage de l'anglais vers le français. En production écrite, les apprenants anglophones de FLE omettent donc souvent ces signes de ponctuation, en particulier la virgule (interférences avec l'anglais).

Les abréviations en anglais américain sont également sources d'erreurs (Dr. Durand au lieu de Dr Durand, Mme. Durand au lieu de Mme Durand, vs. au lieu de vs). Il ne serait pas inutile non plus de préciser aux apprenants de FLE que seules les abréviations pour lesquelles la dernière lettre n'est pas la dernière du mot tronqué sont suivies d'un point, ex. qqch. mais qqn), d'où : $M .<M($ onsieur $)$, mais $D($ octeu $) r, M($ ada)me ou v(ersu)s, excepté pour les unités de mesure telles que $\min$ ou $h(k) m$ ou $(k) g$, etc. (Ramat et Benoit 36, 41).

\subsection{Typographie et chiffres}

La typographie des chiffres est également source d'écueils interférentiels entre l'anglais et le français. Le point en anglais correspondant à une virgule en français et inversement, on rencontre souvent 2.5 au lieu de 2,5 ou 1,200 au lieu de 1.200 ou 1200 . À noter que, pour plus de visibilité, on préfère séparer les tranches de trois chiffres par une espace plutôt que par un point (Goosse et Grevisse 146 ; Perrousseaux 76). Les pourcentages ou les symboles de monnaie sont précédés d'une espace en français (Ramat et Benoit 54), alors qu'en anglais ils sont accolés au chiffre pour les pourcentages et le précèdent pour les symboles de monnaie $(95,5 \%$ en français vs $95.5 \%$ en anglais ; $70 \$$ en français vs $\$ 70$ en anglais).

\subsection{Erreurs courantes liées aux majuscules}

Les écueils interférentiels avec l'anglais relatifs à l'usage des majuscules sont légion. Comme nous allons le montrer, de manière générale, l'anglais utilise beaucoup plus souvent les majuscules que le français (213). Au niveau débutant, on rencontre fréquemment le pronom personnel $J e$ avec une majuscule (même lorsqu'il n'est pas situé en tête de phrase), calqué sur $I$ en anglais. Les noms de jours de la semaine ou de mois portent souvent une majuscule dans les copies des étudiants. Or, contrairement à l'anglais, la minuscule est de rigueur en français, à l'exception des dates historiques (ex. le 11 Septembre). Il en va de même des noms de fête : le Vendredi saint, mais aussi de : Noël, Pâques, etc. et pour les noms de textes sacrés : la Bible, le Coran, la Torah, sans doute parce qu'ils sont uniques. Pour la même raison, on distingue en effet Dieu, dieu unique des religions monothéistes (cf. Mon Dieu!) d'un dieu quelconque (nom commun), la Terre, unique en tant que nom de planète (nom propre) de la terre en tant que nom commun. La majuscule revêt alors un rôle 
lexical distinctif permettant de distinguer un nom propre d'un nom commun. Par exemple, on différencie un hexagone (nom commun), nom de forme géométrique, de l'Hexagone, nom propre désignant la France (unique), de même un état de l'État, une bourse de la Bourse (actions ou valeurs en Bourse). Il ne s'agit alors plus d'un nom commun, mais d'un nom propre, souvent unique et précédé de l'article défini.

Contrairement à Road ou Street, les mots rue, avenue ou boulevard ne prennent pas non plus de majuscule (Ramat et Benoit 85 ; Colignon 105) : l'avenue des Champs Élysées. Plus généralement, pour les toponymes (noms propres géographiques ou de monuments), le nom commun générique est dépourvu de majuscule (seul le terme spécifique, l'élément distinctif, prend la majuscule, Ramat et Benoit 91 ; Colignon 86) : l'ócéan Pacifique, le $\underline{\text { mont }}$ Blanc, l'université Paris VII, la tour Eiffel (vs the Pacific Ocean, Mount Blanc, Paris VII University, the Eiffel $\underline{\text { Tower)}}$. On peut même étendre cette règle à d'autres noms génériques, par exemple, les noms d'organismes d'État multiples (non uniques) comme les ministères : le ministère de l'Éducation nationale (vs the Ministry of National Education ${ }^{4}$ ), les noms de prix : le prix Nobel (vs Nobel Prize) ou les noms de siècles : XVI $I^{e}$ iècle (vs XVI $I^{\text {th }}$ Century).

\subsection{Majuscules et gentilés}

Au début de leur apprentissage, par imitation avec l'anglais, beaucoup d'étudiants mettent aussi systématiquement des majuscules aux gentilés indépendamment de leur catégorie grammaticale, y compris lorsqu'ils sont employés comme adjectifs ou comme noms de langues (adjectifs substantivé) (ex. une chanteuse *Canadienne ; Je parle *Anglais). Or, rappelons que les gentilés ne prennent une majuscule que lorsqu'il s'agit de substantifs, mais prennent une minuscule lorsqu'ils fonctionnent comme des adjectifs. Ainsi, la présence ou non de la majuscule permet de faire la différence entre deux catégories grammaticales : ex. un Parisien, un Européen, un Occidental (substantifs) ; un ordinateur chinois (adjectif) ; le chinois désignant la langue chinoise (adjectif substantivé). On écrira donc un écrivain français, mais un Français. C'est d'ailleurs pourquoi il y aura ou non majuscule selon qu'il s'agira de l'adjectif (il est français) ou de l'attribut considéré comme nom (il est Français), la première solution étant préconisée par l'Académie française (Grevisse et Goosse 110).

\subsection{Autres erreurs liées aux majuscules}

Les noms de religions et de doctrines (ou de courants de pensée) et de leurs adeptes prenant une majuscule en anglais, on rencontre souvent les écueils interférentiels le

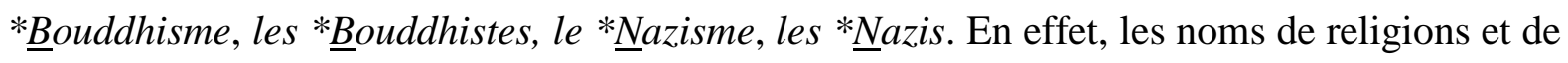
doctrines et de leurs adeptes prennent une minuscule (Callet 58 ; Grevisse et Goosse 111 ; Ramat et Benoit 101) : l'íslam, les musulmans, le gaullisme, les gaullistes. Inversement, les 
noms de groupes à caractéristiques visibles communes (comme la couleur de peau) prennent une majuscule en français et non en anglais (Ramat et Benoit 106). Or, sur les copies de nos élèves, on rencontre souvent les $*_{\underline{n}}$ oirs au lieu de les $\underline{\text { Noirs }}$.

Les noms des points cardinaux font aussi «perdre le nord» aux élèves, car ils prennent une majuscule uniquement «quand ils sont employés seuls (c'est-à-dire sans complément déterminatif) pour désigner une partie du globe, une région d'un continent ou d'un pays » : «le Grand Nord canadien » vs « le nord de la France » (Colignon 95-97). Plus simplement, ils prennent une majuscule lorsqu'ils désignent une zone géographique (la conquête de l'Ouest), mais une minuscule lorsqu'ils désignent une direction (Le vent vient du nord).

Dans le cas des noms d'organismes ou d'institutions uniques, seul le premier terme ainsi que l'adjectif qui le précède prennent une majuscule (Ramat et Benoit 89 ; Perrousseaux 64). On écrira ainsi les Jeux olympiques (vs Olympic Games), la République française,

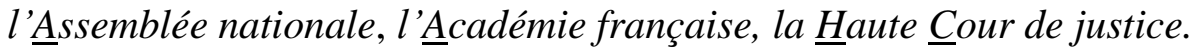

Enfin, contrairement à l'anglais, on ne met pas de majuscule aux noms de fonctions ou titres ou de civilité (Ramat et Benoit 42 ; Colignon 47), même s'il s'agit du pape ou du président d'une nation. On écrira donc le président de la République française, le maire de Grenoble. En revanche, le terme caractérisant une fonction ou un titre porte une majuscule (ce dernier étant le terme spécifique et le nom de la fonction un terme générique) : ex. le ministre de l'Ágriculture. Il en va de même des noms de civilité, madame, monsieur, excepté dans le courrier administratif en tant que marque de déférence (Chère Madame/Cher Monsieur). On écrit majoritairement madame/monsieur sans majuscule lorsqu'on reproduit par écrit des paroles prononcées (Grevisse et Goosse 114 ${ }^{5}$ ). Par ailleurs, les termes d'adresse prennent une majuscule en anglais lorsqu'ils réfèrent à une personne en particulier, comme si on les appelait par leur prénom. Considérés comme des noms propres, ils prennent une majuscule en anglais: Hello, Mum/Dad. Si l'on ne met pas en garde les apprenants concernant cette différence avec l'anglais, ils mettront une majuscule à Maman et Papa en tant que termes d'adresse. Certes, l'usage de la majuscule dans les termes d'adresse n'est pas formellement proscrit, mais il impliquerait une dimension sociolinguistique de déférence (114). On rencontre donc majoritairement le terme d'adresse avec une minuscule dans les dialogues. 


\section{Autres erreurs typographiques}

\subsection{Accents ${ }^{6}$ sur le $e$}

Les accents sont souvent aussi les laissés-pour-compte de l'enseignement et leur explication approfondie absente de la plupart des manuels de FLE. Ils engendrent de

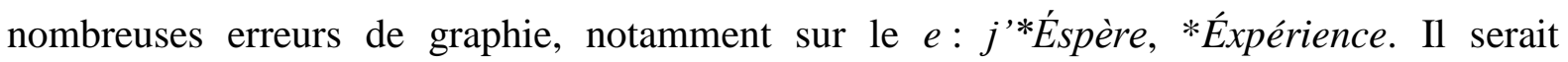
salutaire d'enseigner aux apprenants de FLE qu'un $e$ ne peut pas recevoir un accent lorsqu'il est suivi ${ }^{7}$ de deux consonnes identiques (ex. intElligent) ou différentes (ex. Espoir), sauf si la deuxième est $r$ ou $l$ et la première autre que $r$ ou $l$ (ÉcLairer, $r \underline{E} g L e)^{8}$ et lorsqu'elles représentent un seul son ou phonème, comme les digrammes $g h, c h, p h$, etc., étant alors assimilables à des consonnes simples (rÈgne, pÉchée) (voir l'article de la banque de dépannage linguistique de l'OQLF $^{9}$ ). La lettre $e$ ne porte pas non plus d'accent lorsqu'elle est suivie d'un $x$ (Exercice) - car le graphème $x$ correspond alors à deux phonèmes /gz/ ou /ks/ -, ainsi qu'en fin de mot lorsqu'elle est suivie d'une consonne finale autre que le $s^{10}(a v \underline{E c}$, mang $\underline{E r}$. En revanche, en fin de mot, la lettre $e$ suivie de la consonne finale $s$ s'écrit toujours -Ès avec un accent grave et se prononce [ع] (ex. succ $\underline{\grave{E} s}$, apr $\underline{\grave{E} s}$, Goosse et Grevisse 125-126).

Il se révèle aussi utile de préciser aux apprenants comment savoir si le $e$ accentué reçoit un accent grave ou aigu. Pour ce faire, il existe quelques règles très simples. Comme l'indique l'OQLF dans sa banque de dépannage linguistique ${ }^{11}$, dans un mot dont la syllabe graphique suivante n'est pas muette ${ }^{12}$, la lettre $e$ reçoit un accent aigu (rÉgl(ageler), $c \hat{E} d\left(\right.$ ons/er/ez)). Dans le cas inverse, elle prend un accent grave (ex. rÈglẹ (mentaire ${ }^{13}$ ), discrÈté(ment)), excepté les mots commençant par un e (ex. Épeler, Édredon, sauf ère, Goosse et Grevisse 125), les mots construits avec les préfixes dé- et pré- (ex. dÉtenir, prÉvenir) ${ }^{14}$. C'est pourquoi on rencontre logiquement les oppositions rÉgl(ageler) vs rÈgle(ment), poÈme vs poÉsie. Enfin, la lettre $e$, en fin de mot (comme en début de mot, cf. supra), lorsque le $e$ est prononcé, s'écrit toujours é avec un accent aigu (ex. café, achetÉ $)^{15}$. L'apprenant pourra ainsi, grâce à ces quelques règles ${ }^{16}$, savoir quand un mot est accentué et si l'accent est aigu ou grave.

\section{2. Élisions et traits d'union}

N'oublions pas les élisions obligatoires dont l'anglais est dépourvu. Même au niveau intermédiaire, nos apprenants omettent souvent l'élision : *Je aurais, ...*que on ..., * ${ }^{*} \underline{i}$ il... Ne serait-il alors pas utile d'insister davantage sur la règle selon laquelle la voyelle finale des mots grammaticaux le, la, je, me, te, se, $c e, s i$, que, de et $n e^{17}$ tombe devant un mot commençant par une voyelle ou un $h$ muet, presque constituant une exception (ex. Je suis 
presque arrivé), sauf dans presqu'île? Même si cette règle est enseignée dans les cours de grammaire, elle est rarement retenue ou assimilée, du moins au début de l'apprentissage.

Enfin, terminons par les traits d'union dont les règles ont évolué depuis la réforme orthographique de 1990. Jusque-là, les traits d'union s'utilisaient entre les numéraux cardinaux composés, sauf - le trait d'union étant la « pierre tombale » du et (Fripiat 162) lorsque figurait la conjonction et (ex. vingt et un) ou lorsque l'un des composés était cent ou mille (cent trois, quatre cents, mille trente). Aujourd'hui, ils sont autorisés depuis les rectifications orthographiques de 1990 recommandant leur usage partout. Ainsi, depuis 1990, 103 peut s'écrire cent trois ou cent-trois. En revanche, lorsqu'un pronom personnel et/ou complément est placé derrière le verbe, le trait d'union est toujours requis, que ce soit dans une phrase interrogative lorsqu'on fait l'inversion verbe sujet dans le style formel (Aimes-tu les fraises ?) ou à l'impératif à la forme affirmative, les pronoms suivant aussi le verbe (Disle-moi !, Repose-toi !, Allons-y !). Il serait également bon d'enseigner aux apprenants que la plupart des mots composés sont reliés par des traits d'union (ex. arc-en-ciel, rez-de-chaussée, petit-déjeuner, centre-ville, petit-fils, belle-mère, grand-père), à quelques exceptions près comme petit(e) ami(e).

Face à toutes ces difficultés, l'enseignant devra s'appliquer à montrer comment les pallier et faciliter l'apprentissage en l'intégrant à des objectifs plus vastes de son enseignement, afin de mieux les y ancrer.

\section{Discrimination et remédiation}

\subsection{Sensibilisation à la typographie et à la ponctuation au niveau débutant}

Dès le niveau débutant (niveaux A1 et A2 du CECR), l'enseignant a tout intérêt à sensibiliser les apprenants anglophones à la ponctuation et plus encore à la typographie, notamment à l'usage des majuscules et minuscules. Comme les enseignants du primaire, il peut notamment, dès les premières heures de cours, prendre la bonne habitude d'écrire la date au début de chaque cours (ex. Aujourd'hui, nous sommes le lundi 10 janvier 2016), afin que les apprenants évitent les écueils interférentiels avec l'anglais, liés aux majuscules (lundi et janvier écrits avec une minuscule) ou à la ponctuation (virgule après aujourd'hui, mais absence de virgule avant 2016). Ils intégreraient ainsi plus rapidement les différences de typographie. Comme nous l'avons mentionné dans l'introduction, nous intégrons ces points en les plaçant dans des objectifs plus vastes avec d'autres objectifs tant phonétiques que lexicaux, grammaticaux ou communicatifs, etc. Par exemple, quand nous abordons la famille, les professions et les nationalités, nous enseignons le vocabulaire élémentaire correspondant, 
mais aussi les articles possessifs, les premières phrases et questions pour présenter sa famille. Nous proposons alors plusieurs petits exercices d'application, puis nous demandons aux étudiants de réaliser une fiche de présentation dans laquelle ils doivent faire figurer leur nom, leur nationalité, leur adresse, leur profession, leur langue maternelle ou les langues qu'ils peuvent parler, leur âge, leur date et leur lieu de naissance, leur taille, etc., sans oublier la composition de leur famille. Ce type de fiche de présentation a le mérite de mettre en évidence et d'intégrer certaines différences des règles de typographie et de ponctuation avec l'anglais comme l'élision obligatoire des pronoms je, me ou des articles la/le devant une voyelle ou un $h$ muet, l'absence de virgule devant et dans une énumération ou l'absence de majuscule aux langues. Nous choisissons une ou deux productions que les élèves reproduisent au tableau, ce qui permet aussi une petite initiation à l'écriture cursive tout en soulignant ces différences entre le français et l'anglais.

Je m’appelle X. Je suis coréen(ne). J'habite en Corée, à Séoul. J'étudie le français à l'université $X$.

Ma langue maternelle est le coréen. Je parle le coréen, l'anglais et un peu (le) français.

Je suis né(e) à Séoul le 10 décembre 1990. Je mesure 1,70 m.

Nous sommes cinq dans ma famille: ma sœur, mon frère, mes parents et moi.

Nous recommandons de développer cet exercice à la troisième personne du singulier en prenant comme sujet un autre membre de la famille et surtout un personnage célèbre (ex. Céline Dion).

Lorsque nous abordons les différentes manières de poser des questions, nous en profitons pour souligner la présence d'une espace devant le point d'interrogation, celle de traits d'union dans les questions du type «verbe sujet inversé », le pronom personnel sujet étant placé derrière le verbe (cf. supra), ainsi que l'élision obligatoire du pronom que devant une voyelle (Elle fait quoi? / Que fait-elle? / Qu'est-ce qu'elle fait?). Enseigner les pronoms à l'impératif (à la forme affirmative), les pronoms personnels et/ou complément étant placés également après le verbe, est aussi l'occasion de faire ressortir ces subtilités (Donne-m'en / Va-t'en / Dis-le_lui).

Présenter les fêtes traditionnelles en France est également l'occasion de réduire les erreurs typographiques relatives aux majuscules et virgules superflues dans les dates et aux traits d'union dans les numéros cardinaux (ex. Noël est le vingt-cinq décembre). Lorsque nous enseignons la rédaction des lettres formelles (notamment les lettres formelles demandées à l'épreuve écrite du Delf B2), nous insistons sur la date en en-tête (Strasbourg, 
samedi 26 novembre 2017) et faisons écrire sur des enveloppes l'adresse des étudiants et celle de leur destinataire pour qu'ils prennent mieux conscience de la minuscule à rue, avenue, boulevard ou quai et de la présence d'une virgule après le chiffre $(5$, avenue de la Paix).

Lorsque nous enseignons la civilisation de la France, du Canada ou d'un autre pays francophone, nous en profitons également pour mettre en exergue la typographie des chiffres et des numéros cardinaux (dates, ex. La prise de la Bastille, c'est le 14 juillet 1789 , population, ex. 1500000 habitants; superficie, ex. $555000 \mathrm{~km}^{2}$ ). Plus largement, l'interdisciplinarité est aussi un bon moyen d'enseigner la typographie. Dans cette perspective, nous intégrons à notre enseignement des notions de mathématiques, de géographie, d'histoire, de sciences (chiffres, pourcentage, etc.).

La conjugaison est, quant à elle, un bon exemple pour aborder les accents sur le $e$, notamment l'absence d'accent résultant du redoublement de la consonne. Cette question peut être soulevée par les étudiants curieux, dès le niveau débutant, et ce avant même d'aborder la conjugaison. En effet pourquoi écrire canadien mais canadienne (sans accent sur le $e$ ) ou encore je m'appelle vs nous nous appelons ? Ces deux cas très différents illustrent à leurs manières la nécessité du redoublement de consonne. Cela permet aussi de comprendre que le double $l$ de elles s'appellent n'a rien d'arbitraire ou plus tard d'expliquer et de justifier la conjugaison des verbes du type céder : Je cède vs nous cédons.

\subsection{Ateliers de lecture orale et d'écriture au niveau intermédiaire}

Traiter des problèmes de ponctuation relève aussi de la prosodie. Une des démarches les plus simples pour expliquer l'usage de la virgule est la nécessité de subdivision de la phrase complexe en blocs de sens dans lesquels on doit appliquer une prosodie particulière. Dans cette optique, dans un cours de niveau intermédiaire (niveaux B1 et B2 du CECR, deux séances de quatre-vingt-dix minutes par semaine, soit quarante-cinq heures par semestre), nous demandons aux apprenants de lire chez eux un texte en repérant dans un premier temps uniquement les virgules et de les surligner en bleu, puis dans un deuxième temps de relire le même texte en repérant cette fois-ci les points-virgules, les deux-points et les points et de les surligner en rouge. Ces codes de couleur les aideront à marquer des pauses, courtes pour les virgules en bleu et plus longues pour les signes de ponctuation en rouge. Pour ces exercices de prosodie ou ateliers d'oralisation, nous recourons généralement à un court récit (par exemple une nouvelle, une histoire du Petit Nicolas ou une lettre du roman épistolaire Oscar et la dame rose d'Éric Emmanuel Schmitt) qui présente de nombreux atouts : récit bref contenant à la fois narration et dialogues. Souvent situés en tête de phrase et suivis d'une 
virgule, les compléments circonstanciels et marqueurs temporels ou logiques y sont nombreux (ex. Ce matin, ...; Du coup, ...) et facilitent le repérage et la compréhension de la structure du texte. Enfin, il est demandé aux apprenants d'identifier par une autre couleur les points d'interrogation ou d'exclamation - lesquels indiquent la prosodie de la phrase - afin de penser à changer l'intonation lors de la lecture orale. On parle en effet parfois de «ponctuation mélodique ». Nous consacrons les deux premières séances à la compréhension écrite du texte. En effet, pour une bonne prosodie, les élèves doivent d'abord bien comprendre le texte afin de pouvoir l'oraliser ou le lire différemment selon le contexte (sentiment de tristesse, de colère par exemple). Lors des séances suivantes (dix séances, soit quinze heures de cours), nous formons de petits groupes de quatre à six étudiants selon l'effectif de la classe (classe de vingt à vingt-cinq étudiants) et demandons à chacun d'entre eux de lire ou oraliser en groupe le texte : un élève par personnage pour les parties dialoguées et un ou deux élèves pour la narration. Lors des quatre premières séances, nous circulons de groupe en groupe pour corriger leur prosodie (diction, prononciation, intonation, liaisons, etc.). Puis lors des six séances suivantes, nous leur demandons de «théâtraliser » le texte, autrement dit de le faire vivre devant l'ensemble de la classe en distinguant bien les parties narratives des parties dialoguées et en se souciant des liaisons. Ces exercices de lecture orale se révèlent très efficaces non seulement pour la ponctuation, mais aussi pour la prosodie et la compréhension écrite.

Dans ce même cours, lors des dix-huit dernières séances restantes (soit vingt-sept heures), les ateliers d'écriture sont aussi l'occasion de sensibiliser davantage les apprenants non seulement à la ponctuation, mais aussi à la typographie et à ses différences avec l'anglais (tirets longs suivis d'une espace dans les dialogues pour marquer le changement d'interlocuteur, inversion verbe sujet du verbe introducteur pour rapporter les paroles d'un personnage, espace devant les signes doubles de ponctuation). Dans le cadre de ces ateliers, nous leur faisons toujours en petits groupes (écriture collaborative) soit transposer le court récit théâtralisé lors des dix premières séances, par exemple en en changeant le lieu (dans le pays d'origine de l'apprenant) ou le temps de l'action (à l'heure des technologies mobiles). Les élèves développent ainsi leur créativité, laissant libre cours à leur imagination. Par ailleurs, ces ateliers d'écriture permettent d'apprendre ou de revoir de nombreux points de grammaire (distinction des temps du passé, distinction de l'emploi des articles indéfinis et des articles défini, concordance des temps, pronoms). Chaque groupe relit ensuite à voix haute son texte devant l'ensemble de la classe. Une oralisation ou «théâtralisation » de ces 
récits revisités permet aussi aux auditeurs (autres apprenants) d'améliorer leur compréhension orale.

Toujours au niveau intermédiaire, les ateliers d'écriture et surtout de poésie sont de bons alliés pour apprendre à scinder les syllabes graphiques, ce qui aide non seulement à savoir couper correctement un mot en fin de ligne s'il est manuscrit, mais aussi à savoir quand un mot est accentué ou non sur le $e$ (voir note 8).

\subsection{Corpus parallèles anglais-français et pratique de la traduction au niveau avancé}

Enfin, au niveau avancé (à partir du niveau C1), les corpus parallèles anglais-français sont de bons outils pour mettre en valeur les différences entre l'anglais et le français, aussi bien lexicales que syntaxiques, mais aussi relatives à la typographie et à la ponctuation. Dans cette optique, nous recourons à des textes relativement faciles à lire du type Oscar et la Dame rose d'Éric-Emmanuel Schmitt, à des livres bilingues avec des pages en regard : une page en français, une page en anglais. Ainsi, les apprenants se familiarisent de manière ludique et en contexte avec les différences de typographie et de ponctuation entre le français et l'anglais.

Toujours au niveau avancé, la pratique de la traduction de l'anglais vers le français (thème) est aussi l'occasion de mettre en exergue de nombreux pièges d'interférences, $\mathrm{y}$ compris ceux de typographie et de ponctuation.

\section{Conclusion}

Certes, les erreurs de typographie ou de ponctuation évoquées ici peuvent paraitre secondaires. Cependant, il convient de ne pas en négliger l'importance en les intégrant dans des objectifs plus vastes qui visent aussi bien des compétences langagières, communicatives que culturelles, et, pour en rendre les règles plus accessibles aux apprenants allophones du FLE, nous préconisons de les y sensibiliser dès le niveau débutant pour qu'elles leur semblent aller de soi et que certains automatismes soient ainsi acquis, et ce d'autant plus qu'en raison des nombreuses différences entre le français et l'anglais, ces erreurs de typographie et de ponctuation foisonnent particulièrement chez les apprenants anglophones.

Afin de pallier ou du moins réduire ces écueils interférentiels et autres erreurs typographiques, il s'avère dès lors judicieux et rentable, dès le début de l'apprentissage, soit du niveau débutant jusqu'au niveau avancé, de procéder à diverses activités pour familiariser les apprenants sur ces points tout en intégrant ces derniers dans des cours de FLE. Intégrées dans des objectifs plus vastes, la ponctuation et la typographie s'installent plus naturellement au fil des apprentissages. Au niveau intermédiaire, faire lire un court récit à voix haute (exercices de prosodie), puis le réécrire dans le cadre d'ateliers de lecture orale ou de 
théâtralisation et d'ateliers d'écriture en petits groupes contribue à fixer dans l'esprit des apprenants ces différences entre l'anglais et le français tout en consolidant leurs acquis grammaticaux et lexicaux (enrichissement du vocabulaire) et en favorisant leur créativité et leur autonomie.

Sachant que la ponctuation fournit aux apprenants de FLE la prosodie, aussi bien à travers l'intonation (ponctuation mélodique) qu'à travers le souffle (pauses), elle les aide à la compréhension écrite, mais aussi à la production orale, et partant, à la compréhension orale des autres, en somme à se faire comprendre et donc à communiquer. Comme la ponctuation, la typographie favorise toutes les compétences langagières, y compris la production écrite et même la compétence communicative, qui est l'objectif principal de tout apprenant d'une langue étrangère. En somme, intégrer ponctuation et typographie à l'enseignement d'une langue étrangère à travers une approche globale se révèle non pas secondaire, mais au contraire essentiel.

\section{Bibliographie}

Ballard, Michel. Versus : la version réfléchie. Repérages et paramètres. Paris : Ophrys, 2003. Callet, Stéphanie. Répertoire des difficultés du français. Vérifier, comprendre, appliquer. Grenoble : PU de Grenoble, 2013.

Colignon, Jean-Pierre. La majuscule, c'est capital! Paris : Albin Michel, 2005.

Demanuelli, Claude. Points de repère: Approche interlinguistique de la ponctuation français-anglais. Saint Etienne : CIEREC, 1987.

Fripiat, Bernard. L'orthographe 99 trucs pour en rire et la retenir! Dole : Gunten, 2013.

Grevisse, Maurice et André Goosse. Le Bon Usage. $13^{\mathrm{e}}$ édition. Bruxelles: De Boeck/Duculot, 2001.

Han, Jeon-gil. «An Analysis of Korean Student's Errors in French Composition : English Inference ». Linguistic Research 25.1 (2008) : 103-125.

Ilani, Rozita. «Carrefour de l'apprentissage des langues : le persan, l'anglais et le français ». Voix plurielles 13.1 (2016) : 31-42.

Kellerman, Éric. « Eil pour œil ». Encrages, $n^{\circ}$ spécial, Acquisition d’une langue étrangère, (1980) : 54-63.

Merieux, Régine et Yves Loiseau. Connexions, niveau 2. Paris : Didier, 2004.

Narjoux, Cécile. La ponctuation. Bruxelles : De Boeck-Duculot, 2010.

Perrousseaux, Yves. Règles typographiques de l'orthographe du français. Paris: Ateliers Perrouseaux, 2010. 
Ramat, Aurel et Anne-Marie Benoit. Le Ramat de la typographie. Montréal : Anne-Marie Benoit éditrice, 2012.

Riegel, Martin, Jean-Christophe Pellat et René Rioul. Grammaire méthodique du français. Paris : PUF, 2009.

Védénina, Ludmila. Pertinence linguistique de la disposition typographique. Paris-Bruxelles : Peeters Selaf, 1989.

Notes
${ }^{1}$ Certains auteurs emploient le terme de «ponctuation » dans un sens plus large, incluant, à l'instar de Ludmila
Védénina (141), «certains procédés graphiques comme l'emploi des caractères, de l'espace blanc entre les
signes, etc. ». Martin Riegel, Jean-Christophe Pellat et René Rioul (141) considèrent l'espace comme un signe
" en négatif ». Dans cet article, nous ne préciserons pas si l'espace est insécable ou sécable et ne distinguerons
pas les espaces fines des espaces fortes.
2 Pour les apprenants asiatiques, les difficultés sont d'autant plus complexes que leurs erreurs résultent à la fois
d'interférences avec l'anglais et leur LM. À titre d'exemple, leur LM ne mettant ni d'espace après les chiffres,
ni devant les parenthèses ou les crochets, ils accolent souvent les chiffres au mot qui suit ( $J$ 'ai *20ans) ou aux
parenthèses : *CP(Cours Préparatoire). Une étude plus approfondie sur les erreurs de ponctuation et de
typographie spécifiques aux apprenants asiatiques nécessiterait une étude à part entière.
${ }^{3}$ Les normes des règles typographiques peuvent légèrement varier dans d'autres pays francophones comme au
Canada, notamment s'agissant des signes doubles de ponctuation, appelées aussi «ponctuations hautes » (;: ? !)
(Ramat et Benoit 121 , tableau détaillé page 191). En effet, au Canada, devant ces signes doubles de ponctuation,
figure soit une espace fine insécable, soit aucune. Cependant, nos cours sont conçus pour des apprenants visant
des diplômes FLE ou équivalents pensés par le CIEP (donc aux standards français).
${ }^{4}$ bdl.oqlf.gouv.qc.ca/bdl/gabarit_bdl.asp?id=1289, consulté le 20.11 .2017 .
${ }^{5}$ bdl.oqlf.gouv.qc.ca/bdl/gabarit_bdl.asp?id=3515, consulté le 28.11 .2017 .
${ }^{6}$ Comme le précise l'OQLF, les accents sont apparus tardivement dans la langue française afin de préciser la
prononciation des voyelles (bdl.oqlf.gouv.qc.ca/bdl/gabarit_bdl.asp?Th=2\&tl=\&id=3692, consulté le 20.11.2017).

${ }^{7}$ Notons que dans certains mots préfixés ou composés, généralement de formation récente, les deux éléments formants continuent à être perçus chacun avec leur signification propre, indépendamment de la coupe syllabique, et le $e$ conserve sa graphie initiale (Goosse et Grevisse 125) : soit il reçoit un accent aigu sur le $e$, même si celuici est suivi de deux consonnes différentes ( + consonne), notamment avec les préfixes dé- et pré- ou l'élément formant télé- (ex. dÉ/stabiliser, prÉ/scolaire, télÉ/spectateur), soit il est non accentué sur le e lorsqu'il est terminé par un -e- suivi d'une consonne finale (ex. hypEr/activité, intEr/action).

${ }^{8}$ À l'instar de l'OQLF (voir note 9), nous préférons enseigner cette règle, plutôt que celle donnée par la plupart des grammaires (voir Grevisse et Goosse 125) ou des sites Internet (www.aidenet.eu/nouveau04.htm, consulté le 20.11.2017), à savoir : la lettre $e$ n'est accentuée que si elle est en fin de syllabe graphique, sauf devant un $s$ final (ex. $m E ́ / t r o$ vs $m E r / c i$, in/tÉ/ressant vs in/tEl/ligent, $v \hat{E} /$ te/ment vs $v E s / t e$ ). Il faudrait néanmoins alors savoir couper les syllabes graphiques d'un mot (voir Grevisse et Goosse 31 ; Benoit et Ramat 112-113). Les règles de la syllabation graphique gagneraient néanmoins à être enseignées au niveau avancé pour l'écriture manuscrite. Elles permettraient non seulement de couper correctement un mot en fin de ligne (l'anglais autorisant la coupure de mots en milieu de syllabe), mais aussi une fois assimilées, faciliteraient la règle des accents sur le $e$. De surcroît, elles ont en commun les exceptions des groupes de deux consonnes qui représentent un seul son ou phonème et celles où la deuxième est $r$ ou $l$ (voir note 9) lorsque la première n'est ni $r$ ni $l$. En effet, excepté dans ces deux cas, lorsqu'on a deux consonnes entre deux voyelles, on coupe entre les deux consonnes; si on a trois consonnes (ou plus), on coupe après la deuxième (ex. obs-/tacle).

${ }^{9}$ «Dans [le] contexte [où le élè termine la syllabe graphique], le élè peut être suivi d'une consonne simple, de deux consonnes qui représentent un seul son ou encore d'un groupe de deux consonnes se terminant par un $l$ ou un $\quad r \gg ; \quad$ accent aigu bdl.oqlf.gouv.qc.ca/bdl/gabarit_bdl.asp?id=3693, accent grave bdl.oqlf.gouv.qc.ca/bdl/gabarit_bdl.asp?Th=2\&t1=\&id=3694, consulté le 20.11.2017.

10 Excepté dans quelques mots en -êt (intér $\hat{E} t$, arr $\hat{E} t$, prêt, for $\hat{E} t$ ) où l'accent circonflexe est un vestige du $s$ (cf. en anglais interest, arrest, forest), ainsi que quelques noms propres en -ël où -el est précédé d'une voyelle (ex. Noël, Israël).

${ }^{11}$ bdl.oqlf.gouv.qc.ca/bdl/gabarit_bdl.asp?t1=1\&id=3695, consulté le 20.11.2017.

12 Une syllabe muette contient exclusivement un $e$ muet ou un e prononcé [ə] (ex. règle, règlement). 
13 C'est pourquoi, la réforme de 1990 a rectifié les quelques rares anomalies, initialement écrites avec un accent aigu, conformément à cette règle et à leur véritable prononciation : évÈnement, crÈmerie, cÈleri, sÈcheresse, ass Èchement, rÈglement(er/airelation), etc., ainsi que dans les inversions interrogatives avec la première personne du singulier en $e$ suivie du pronom sujet je (ex. puiss $\grave{E}-j e$ ). De même, la conjugaison des verbes du premier groupe dont l'avant-dernière syllabe se termine à l'infinitif par un é (céder, régler, altérer, alléger, etc.) s'est, depuis la réforme de 1990, alignée sur celle du verbe semer et ces verbes ne maintiennent plus, au futur et au conditionnel présent, l'accent aigu de l'infinitif : ex. je rÈglerai, je rÈglerais (pour plus de détails, voir Benoit et Ramat 182 et les liens www.gqmnf.org et bdl.oqlf.gouv.qc.ca/bdl/gabarit_bdl.asp?id=3165, consultés le 20.11.2017.

14 Y compris lorsque le é est suivi de la marque du pluriel et/ou d'un e muet (ex. cafÉs, lycÉe).

15 Prennent également un accent aigu $m E ́ d e c i n(e)$ et les mots composés contenant l'élément formant télé(téléreportage) (bdl.oqlf.gouv.qc.ca/bdl/gabarit_bdl.asp?id=3165, consulté le 20.11.2017).

16 Pour expliquer les accents sur le $e$, nous recommandons la vidéo de Home Language : www.youtube.com/watch?v=MbryaGRY7eA\&list=PLB2vxd8Tj1cXOt8-RDvBXQG4TWvTdBXfm\&index=5, consulté le 21 novembre 2017.

${ }^{17}$ Pour plus de précisions, voir bdl.oqlf.gouv.qc.ca/bdl/gabarit_bdl.asp?id=1737. 